\title{
Metabolische und reproduktionsmedizinische Aspekte des Polyzystischen Ovarsyndroms (PCOS)
}

\author{
Michael Feichtinger · Tina Stopp · Christian Göbl
}

Online publiziert: 27. Januar 2016

(C) Die Autor(en) 2016. Dieser Artikel ist auf Springerlink.com mit Open Access verfügbar.

\begin{abstract}
Zusammenfassung Das polyzystische Ovarsyndrom ist die häufigste endokrine Störung von Frauen im reproduktiven Alter. Symptome beinhalten metabolische, gynäkologische und kosmetische Aspekte. Genetische Faktoren leisten einen großen Beitrag zur Manifestation der Erkrankung. So haben männliche Verwandte von PCOS Patientinnen ähnliche Symptome. Eine zentrale Rolle spielen Lebensstilfaktoren, die die klinische Erscheinung des Syndroms wesentlich beeinflussen. Im folgenden Artikel soll ein Überblick über Epidemiologie, metabolische und gynäkologische Aspekte, gender- und genetische Faktoren sowie Therapie von PCOS geliefert werden.
\end{abstract}

Schluisselwörter Polyzystisches Ovarsyndrom • Stoffwechsel $\cdot$ Reproduktionsmedizin

\section{Metabolic and Reproductive Consequences of the Polycystic Ovary Syndrome (PCOS)}

Summary Polycystic ovarian syndrome represents the most common endocrine disease of women of reproductive age. Symptoms include metabolic, gynecologic and cosmetic features. Genetic factors seem to contribute to the disease, affecting not only women but also male relatives of patients with similar symptoms.

C. Göbl, MD, PhD, MSc ( $\square) \cdot$ M. Feichtinger, MD · T. Stopp, MBA Abteilung für gynäkologische Endokrinologie und

Reproduktionsmedizin, Universitätsklinik für Frauenheilkunde,

Währinger Gürtel 18-20,

1090 Wien, Österreich

E-Mail: christian.goebl@meduniwien.ac.at

M. Feichtinger, MD · T. Stopp, MBA

Wunschbaby Institut Feichtinger,

Lainzerstraße 6,

1130 Wien, Österreich
Besides, lifestyle factors play a central role impacting clinical PCOS appearance. Following we present an overview of the syndrome, its epidemiology, metabolic and gynecological aspects, gender and genetic factors and its therapy.

Keywords Polycystic Ovarian Syndrome - Metabolism • Reproductive Medicine

\section{Epidemiologie und Klassifizierung}

Das polyzystische Ovarsyndrom (PCOS) ist die häufigste endokrine Störung von Frauen im reproduktionsfähigem Alter. Je nach Klassifizierung sind zwischen 6 und 19,9\% aller Frauen betroffen [1,2]. Bereits Hippokrates (460 - 377 v.c.) hat ein ähnliches Krankheitsbild beschrieben [3]. Zu den typischen klinischen Symptomen zählen Hirsutismus, Oligo- oder Amenorrhoe, Akne, Alopezie und Infertilität. Gleichzeitig besteht bei fast zwei Drittel eine metabolische Störung (in Form von Insulinresistenz und kompensatorischer Hyperinsulinämie), die das Risiko für Diabetes Mellitus und Herz-Kreislauf-Erkrankungen stark erhöht [4].

Zur Diagnosesicherung werden je nach Klassifikation unterschiedliche Konstellationen von Oligobzw. Anovulation, klinischer oder biochemischer Hyperandrogenämie und polyzystischen Ovarien im Ultraschall herangezogen (Tab. 1). Dabei wird der Hyperandrogenämie zunehmende Bedeutung beigemessen [5-7].

\section{Metabolische Aspekte}

Eine Assoziation der Erkrankung mit Übergewicht und metabolischen Störungen gilt als gesichert [8]. In bis zu $40 \%$ der PCOS Patientinnen zeigen im Alter von 30-40 
Tab. 1 Diagnosekriterien nach der Rotterdam und NIH Klassifikation modifiziert nach Azziz et al. [6]

\begin{tabular}{|l|l|l|l|l|}
\hline Hyperandrogenämie & Oligo/Anovulation & PCO-Ovarien & NIH & Rotterdam \\
\hline$\sqrt{ }$ & $\sqrt{ }$ & $\sqrt{ }$ & $\sqrt{ }$ & $\sqrt{ }$ \\
\hline$\sqrt{ }$ & $\sqrt{ }$ & - & $\sqrt{ }$ & $\sqrt{ }$ \\
\hline$\sqrt{ }$ & - & $\sqrt{ }$ & - & $\sqrt{ }$ \\
\hline- & $\sqrt{ }$ & $\sqrt{ }$ & - & $\sqrt{ }$ \\
\hline
\end{tabular}

Jahren eine gestörte Glukosetoleranz oder Typ 2 Diabetes $[9,10]$. Bei adipösen PCOS Patientinnen kann sogar in bis zu $95 \%$ eine ausgeprägte Insulinresistenz festgestellt werden [11]. Das PCO-Syndrom scheint hierbei einen eigenständigen Risikofaktor darzustellen, da sich die hohe Rate an Glukosestoffwechselstörungen nicht alleine mit der erhöhten Prävalenz von Übergewicht und Adipositas erklären lässt [12]. Insgesamt zeigten PCOS Patientinnen ein 5-fach erhöhtes Risiko, innerhalb eines 8-jährigen Beobachtungszeitraumes an Diabetes zu erkranken [13]. Zusätzlich haben PCOS Patientinnen ein zweifach erhöhtes Risiko für das Auftreten eines „metabolischen Syndroms" sowie einer Dyslipidämie [14, 15]. Weiters wurde eine erhöhte Inzidenz für koronare Gefäßverkalkungen gezeigt [16].

\section{Geburtshilfliche und gynäkologische Aspekte}

Es wird vermutet, dass hinter der Pathogenese des PCOS eine ovarielle Hyperandrogenämie und eine durch Insulinresistenz hervorgerufene Hyperinsulinämie stehen, die gemeinsam mit parakrinen Signalen im Ovar zu einer Hemmung des Follikelwachstums führen [17]. Selbst PCOS Patientinnen, die über einen regelmäßigen Zyklus berichten, haben in $40 \%$ keine Ovulation [18], weshalb PCOS Patientinnen oft die assistierte Reproduktion benötigen, um eine Schwangerschaft $\mathrm{zu}$ erreichen. Jedoch sind die gewonnenen Eizellen von PCOS Patientinnen oft von schlechter Qualität; möglicherweise kommt es hierbei $\mathrm{zu}$ einer fehlenden Komplettierung der Meiose [19], wobei sich speziell die Hyperandrogenämie negativ auszuwirken scheint [20].

PCOS Patientinnen erleiden vermehrt Aborte [21]; auch vermehrte Schwangerschaftskomplikationen wie Gestationsdiabetes, Präeklampsie und Frühgeburten wurden bei schwangeren PCOS Patientinnen beobachtet [22]. Speziell Gestationsdiabetes - hervorgerufen durch eine präkonzeptionell vorbestehende Insulinresistenz, die sich im Laufe der Schwangerschaft verschlechtert kann in weiterer Folge zu schweren geburtshilflichen Komplikationen führen, welchen jedoch mit einem oralen Glukosetoleranztest (oGTT) und somit frühen Diagnose durch eine entsprechende Therapie vorgebeugt werden kann [23]. Aufgrund dieses erhöhten Risikos bei PCOS Patientinnen scheint somit ein Screening in der Frühschwangerschaft mittels Nüchternblutzucker, HbAlc und eventuell frühem oGTT indiziert (sollte die
Abklärung des Glukosestoffwechsels nicht bereits präkonzeptionell durchgeführt worden sein) [24].

Mit fortgeschrittenem Alter werden durch ein Absinken der ovariellen Reserve weniger Androgene gebildet und es kommt zu regelmäßigeren Zyklen und verminderten Symptomen der Hyperandrogenämie [25, 26]. Gleichzeitig spielen bei zunehmendem Alter die erhöhte Insulinresistenz und der steigende BMI eine zunehmende Rolle [27].

Patientinnen mit PCOS haben ein erhöhtes Risiko an Endometriumkarzinom zu erkranken; auch ein erhöhtes Risiko für Brust- und Ovarialkarzinom wird diskutiert [28].

\section{Gender und genetische Faktoren}

Mehrere Studien konnten eine familiäre Häufung von PCOS beobachten.

So leiden $20-40 \%$ der weiblichen Verwandten ersten Grades von PCOS Patientinnen ebenfalls an der Erkrankung [29]. Auch männliche Verwandte von PCOS Patientinnen zeigen erhöhte Werte an DHEAS sowie ein höheres metabolisches Risiko [30-32]. Darüber hinaus kommt es bei männlichen Nachkommen von PCOS Patientinnen zu einem früheren Auftreten von Stoffwechselstörungen [33]. Andererseits wurde bei Vätern von PCOS Patientinnen eine erhöhte Inzidenz von HerzKreislauf-Erkrankungen festgestellt [34].

\section{Therapie}

Die Behandlung der PCOS Symptome umfasst meist eine Östrogen-Progesteron Pille sowie bei ausgeprägten Formen der Hyperandrogenämie eine Androgenblockade (Cyproteronacetat, Spironolacton, Finasterid). Gleichzeitig können mit kosmetischen Behandlungsmethoden wie mechanischer Haarentfernung mit Laser und Bleichungen gute Resultate erzielt werden. Eine zentrale Rolle spielt jedoch eine Änderung des Lebensstils, mit körperlicher Bewegung und Gewichtsabnahme [35]. Insgesamt wurden sehr unterschiedliche Ausprägungen von PCOS je nach Lebensstil beobachtet. So zeigen betroffene Frauen in den USA ausgeprägtere Symptome als Frauen in Europa [36]. Speziell bei übergewichtigen Frauen mit gestörter Glukosetoleranz sollte eine Therapie mit Metformin erwogen werden [37]. Hierbei wird die periphere Insulinsensitivität erhöht und somit einer chronischen Hyperinsulinämie vorgebeugt [38]. In weiterer Folge kommt es zu einem schnelleren Gewichtsverlust und bei vielen Frauen zeigt sich ein erneut einsetzender regelmäßiger Menstruationszyklus [37].

Bei Kinderwunsch kann langfristig eine Therapie mit Metformin zu erhöhten Schwangerschaftsraten führen, bei dringendem Kinderwunsch sind jedoch Clomiphen und Letrozol das Mittel erster Wahl [38-40]. 


\section{Open Access}

Dieser Artikel unterliegt den Bedingungen der Creative Commons Attribution License. Dadurch sind die Nutzung, Verteilung und Reproduktion erlaubt, sofern der/die Originalautor/en und die Quelle angegeben sind.

\section{Einhaltung ethischer Richtlinien}

\section{Interessenkonflikt}

M. Feichtinger, T. Stopp und C. Göbl geben an, dass kein Interessenkonflikt besteht.

Dieser Beitrag beinhaltet keine Studien an Menschen oder Tieren.

\section{Literatur}

1. March WA, Moore VM, Willson KJ, et al. The prevalence of polycystic ovary syndrome in a community sample assessed under contrasting diagnostic criteria. Human Reprod. 2010;25:544-51.

2. Yildiz BO, Bozdag G, Yapici Z, et al. Prevalence, phenotype and cardiometabolic risk of polycystic ovary syndrome under different diagnostic criteria. Hum Reprod. 2012;27:3067-73.

3. Azziz R, Dumesic DA, Goodarzi MO. Polycystic ovary syndrome: an ancient disorder? Fertil Steril. 2011;95:1544-8.

4. Goodarzi MO, Dumesic DA, Chazenbalk G, et al. Polycystic ovary syndrome: etiology, pathogenesis and diagnosis. Nat Rev Endocrinol. 2011;7:219-31.

5. Rotterdam EA-SPcwg. Revised 2003 consensus on diagnostic criteria and long-term health risks related to polycystic ovary syndrome (PCOS). Hum Reprod. 2004;19:41-7.

6. Azziz R, Carmina E, Dewailly D, et al. Positions statement: criteria for defining polycystic ovary syndrome as a predominantly hyperandrogenic syndrome: an Androgen Excess Society guideline. J Clin Endocrinol Metab. 2006;91:4237-45.

7. Okoroh EM, Hooper WC, Atrash HK, et al. Prevalence of polycystic ovary syndrome among the privately insured, United States, 2003-2008. Am J Obstet Gynecol. 2012;207:299 e1-7.

8. Wilson JF. In the clinic. The polycystic ovary syndrome. Ann Intern Med. 2011;154:ITC-2-ITC-15. (quiz ITC2-6).

9. Norman RJ, Masters L, Milner CR, et al. Relative risk of conversion from normoglycaemia to impaired glucose tolerance or non-insulin dependent diabetes mellitus in polycystic ovarian syndrome. Hum Reprod. 2001;16:1995-8.

10. Moran LJ, Misso ML, Wild RA, et al. Impaired glucose tolerance, type 2 diabetes and metabolic syndrome in polycystic ovary syndrome: a systematic review and metaanalysis. Hum Reprod Update. 2010;16:347-63.

11. Carmina E, Lobo RA. Use of fasting blood to assess the prevalence of insulin resistance in women with polycystic ovary syndrome. Fertil Steril. 2004;82:661-5.

12. DeUgarte CM, Bartolucci AA, Azziz R. Prevalence of insulin resistance in the polycystic ovary syndrome using the homeostasis model assessment. Fertil Steril. 2005;83:1454-60.

13. Boudreaux MY, Talbott EO, Kip KE, et al. Risk of T2DM and impaired fasting glucose among PCOS subjects: results of an 8-year follow-up. Curr Diab Rep. 2006;6:77-83.
14. Apridonidze T, Essah PA, Iuorno MJ, et al. Prevalence and characteristics of the metabolic syndrome in women with polycystic ovary syndrome. J Clin Endocrinol Metab. 2005;90:1929-35.

15. Rocha MP, Marcondes JA, Barcellos CR, et al. Dyslipidemia in women with polycystic ovary syndrome: incidence, pattern and predictors. Gynecol Endocrinol. 2011;27:814-9.

16. Talbott EO, Zborowski JV, Rager JR, et al. Evidence for an association between metabolic cardiovascular syndrome and coronary and aortic calcification among women with polycystic ovary syndrome. J Clin Endocrinol Metab. 2004;89:5454-61.

17. Gougeon A. Regulation of ovarian follicular development in primates: facts and hypotheses. Endocr Rev. 1996;17:121-55.

18. Azziz R, Waggoner WT, Ochoa T, et al. Idiopathic hirsutism: an uncommon cause of hirsutism in Alabama. Fertil Steril. 1998;70:274-8.

19. Schramm RD, Bavister BD. A macaque model for studying mechanisms controlling oocyte development and maturation in human and non-human primates. Hum Reprod. 1999;14:2544-55.

20. Cano F, Garcia-Velasco JA, Millet A, et al. Oocyte quality in polycystic ovaries revisited: identification of a particular subgroup of women. J Assist Reprod Genet. 1997;14:254-61.

21. Tian L, Shen H, Lu Q, et al. Insulin resistance increases the risk of spontaneous abortion after assisted reproduction technology treatment. J Clin Endocrinol Metab. 2007;92:1430-3.

22. Boomsma CM, Eijkemans MJ, Hughes EG, et al. A metaanalysis of pregnancy outcomes in women with polycystic ovary syndrome. Hum Reprod Update. 2006;12:673-83.

23. HAPO Study Cooperative Research Group, Metzger BE, Lowe LP, et al. Hyperglycemia and adverse pregnancy outcomes. N Engl J Med. 2008;358(19):1991-2002.

24. American Diabetes Association. (12) management of diabetes in pregnancy. Diabetes Care. 2015;38 Suppl:S77-9.

25. Elting MW, Korsen TJ, Rekers-Mombarg LT, et al. Women with polycystic ovary syndrome gain regular menstrual cycles when ageing. Hum Reprod. 2000;15:24-8.

26. Bili H, Laven J, Imani B, et al. Age-related differences in features associated with polycystic ovary syndrome in normogonadotrophic oligo-amenorrhoeic infertile women of reproductive years. Eur J Endocrinol. 2001;145:749-55.

27. Panidis D, Tziomalos K, Macut D, et al. Cross-sectional analysis of the effects of age on the hormonal, metabolic, and ultrasonographic features and the prevalence of the different phenotypes of polycystic ovary syndrome. Fertil Steril. 2012;97:494-500.

28. Dumesic DA, Lobo RA. Cancer risk and PCOS. Steroids. 2013;78:782-5.

29. Kahsar-Miller MD, Nixon C, Boots LR, et al. Prevalence of polycystic ovary syndrome (PCOS) in first-degree relatives of patients with PCOS. Fertil Steril. 2001;75:53-8.

30. Legro RS, Kunselman AR, Demers L, et al. Elevated dehydroepiandrosterone sulfate levels as the reproductive phenotype in the brothers of women with polycystic ovary syndrome. J Clin Endocrinol Metab. 2002;87:2134-8.

31. Kaushal R, Parchure N, Bano G, et al. Insulin resistance and endothelial dysfunction in the brothers of Indian subcontinent Asian women with polycystic ovaries. Clin Endocrinol (Oxf). 2004;60:322-8.

32. Coviello AD, Sam S, Legro RS, et al. High prevalence of metabolic syndrome in first-degree male relatives of women with polycystic ovary syndrome is related to high rates of obesity. J Clin Endocrinol Metab. 2009;94:4361-6. 
33. Recabarren SE, Smith R, Rios R, et al. Metabolic profile in sons of women with polycystic ovary syndrome. J Clin Endocrinol Metab. 2008;93:1820-6.

34. Taylor MC, Reema Kar A, Kunselman AR, et al. Evidence for increased cardiovascular events in the fathers but not mothers of women with polycystic ovary syndrome. Hum Reprod. 2011;26:2226-31.

35. Bruner B, Chad K, Chizen D. Effects of exercise and nutritional counseling in women with polycystic ovary syndrome. Appl Physiol Nutr Metab. 2006;31:384-91.

36. Carmina E, Legro RS, Stamets $K$, et al. Difference in body weight between American and Italian women with polycystic ovary syndrome: influence of the diet. Hum Reprod. 2003;18:2289-93.

37. Nestler JE. Metformin for the treatment of the polycystic ovary syndrome. N Engl J Med. 2008;358:47-54.
38. Antonucci $\mathrm{T}$, Whitcomb $\mathrm{R}$, McLain $\mathrm{R}$, et al. Impaired glucose tolerance is normalized by treatment with the thiazolidinedione troglitazone. Diabetes Care. 1997;20:188-93.

39. Palomba S, Pasquali R, Orio F, et al. Clomiphene citrate, metformin or both as first-step approach in treating anovulatory infertility in patients with polycystic ovary syndrome (PCOS): a systematic review of head-to-head randomized controlled studies and meta-analysis. Clin Endocrinol (Oxf). 2009;70:311-21.

40. Legro RS, Brzyski RG, Diamond MP, et al. Letrozole versus clomiphene for infertility in the polycystic ovary syndrome. N Engl J Med. 2014;371:119-29. 